\title{
Is pain in the brain?
}

\author{
Geraint Rees* and Sarah Edwards \\ University College London Institute of Cognitive Neuroscience, 17 Queen Square, London WC1N \\ 3AR UK
}

\section{Summary}

There has been much recent interest in pioneering neuroimaging experiments studying brain responses to sensory stimulation in patients in minimally conscious and persistent vegetative states. Here, we discuss recent work by Melanie Boly and her colleagues, in which brain responses to stimulation of the median nerve were measured by use of PET in healthy volunteers and in patients in either minimally conscious or persistent vegetative state. The stimulation was rated painful by the volunteers, but did not elicit behavioral responses in either of the two patient groups. Nevertheless, brain activation in patients in a minimally conscious (but not persistent vegetative) state was seen in areas very similar to those activated by the stimuli judged painful in healthy volunteers. Here, we discuss whether such data can be used to conclude that individuals with severe brain damage feel pain, and consider the ethically appropriate clinical response to such important new data.

\section{Keywords}

pain; minimally conscious state; functional imaging; PET

\section{Commentary}

Doctors elicit the thoughts and feelings of their patients by taking a history. This verbal (and nonverbal) communication with a patient remains central to making a diagnosis and monitoring symptoms such as pain and distress. We do not yet fully understand what underlying patterns of brain activity encode these conscious experiences. Such an understanding could allow these patterns to be decoded and used to predict perceptions and intentions without direct communication.

In recent years, there have been considerable advances in the use of noninvasive measures of neural activity to predict perceptions and intentions in healthy individuals. 1 While somewhat limited in their scope, these early reports raise the intriguing possibility that such approaches might be useful in clinical situations where communication with patients is difficult or impossible. As reported in Lancet Neurology, Boly and colleagues 2 have addressed this possibility by studying brain responses to noxious stimuli in patients in a minimally conscious state (MCS). Their findings raise intriguing and important ethical and scientific questions concerning pain perception in individuals with severe brain damage.

MCS is a condition of dramatically altered consciousness in which minimal but definite behavioral evidence of self or environmental awareness is demonstrated.3 Patients in MCS cannot communicate, and behavioral evidence of awareness can be inconsistent and difficult

*Correspondence University College London Institute of Cognitive Neuroscience, 17 Queen Square, London WC1N 3AR UK g.rees@fil.ion.ucl.ac.uk. 
to demonstrate. Using PET imaging, Boly and colleagues showed that in both patients in MCS and healthy controls, noxious stimulation of the median nerve activated similar regions of the thalamus, primary and secondary somatosensory cortices, and the frontoparietal and anterior cingulate cortex. Activity in these areas—known as the 'pain matrix'-plus coupling between primary sensory areas and frontoparietal association cortex was markedly greater in patients in MCS than in patients in persistent vegetative state, who show no evidence of self or environmental awareness. Such coupling is hypothesized to be important in mediating awareness.4

Clinically, can we conclude from these patterns of brain activity that patients in MCS feel pain? This question has both scientific and ethical dimensions. From a scientific standpoint, it would be premature to reach such a strong conclusion. The authors observed some differences in the quantity and quality of activation in the pain matrix when comparing MCS and healthy volunteers. This finding is difficult to interpret, because we do not yet know precisely which areas (and what levels of activity) are both necessary and sufficient to elicit pain perception in healthy individuals. Furthermore, it is important to remember that the individuals in MCS had severe brain damage, and cannot, therefore, be regarded as being just like healthy controls, apart from an inability to report their experiences verbally.

The patients in MCS did not exhibit behavioral responses to noxious stimuli; in other words, they showed a dissociation between brain activity (present) and behavior (absent). Such dissociations typically prompt quite different conclusions in other types of brain injury. In individuals with parietal neglect, for example, responses to visual stimuli can be identified in regions of visual cortex corresponding to those activated in healthy volunteers, even though the patients cannot report these stimuli.5 However, we do not conclude from the similar patterns of brain responses that patients with neglect are actually aware of these stimuli. We cannot yet conclude, therefore, that patients in MCS have an inner mental life, including the conscious perception of pain. The presence of brain activation is insufficient evidence for the perception of pain unless it can be shown that the same activation cannot occur in the absence of pain.6

For clinicians, there is the immediate practical question of whether or not to assume that patients in MCS can feel pain, and this takes us into the realm of ethics. It is far from obvious how the interests of patients in MCS might best be served - ethical questions of withholding or withdrawing artificial life support tend to focus on the patient's complete loss of functioning and inability to be restored to anything like a fulfilling and happy life rather than on their capacity to feel pain. Pain might, however, be an inevitable-if unintendedbyproduct of keeping such patients alive.7 Nevertheless, few would disagree that steps should be taken to avoid pain, provided that they do not jeopardize otherwise beneficial treatment.

Once the decision has been made to initiate or continue artificial life support, it would seem intuitively sensible to treat the patient for pain, just in case they are able to experience it despite being minimally conscious. This is a somewhat unorthodox application of the 'precautionary principle', which is used in situations of genuine uncertainty or ambiguity where any harm would be severe or irreversible. 8 There are several formulations of this principle, but if we choose to accept it, the burden of proof should always be on those claiming that there is no harm done-in this case, those arguing that patients in MCS are incapable of feeling pain if left untreated. We might tentatively propose that there is now reason to believe that patients in MCS experience pain to such an extent that the clinical use of analgesics could be considered a moral requirement. There could be questions of financial cost, but we would not expect any additional costs of the analgesics on top of the lifesupport facility to be a pressing concern. 
Boly et al. have presented important new evidence in a challenging area of medical practice. While scientifically falling short of demonstrating that patients in MCS have first-person experiences such as pain, this study should prompt more research in this vitally important area. Moreover, clinicians making practical decisions about how to treat such patients may need to take a cautious approach, and to use analgesics unless sufficient evidence accumulates to indicate that such patients do not experience pain.

\section{Practice Point}

Noxious stimulation results in activation of similar brain areas in healthy volunteers and patients in a minimally conscious state (MCS), although this finding does not definitively demonstrate conscious pain perception in patients in MCS. Nevertheless, clinicians should continue to treat individuals in MCS with analgesics where clinically appropriate, just in case they do feel pain.

\section{Biography}

Biographies

G Rees is a Professor of Cognitive Neurology at the University College London (UCL) Institute of Cognitive Neuroscience and Wellcome Trust Centre for Neuroimaging at UCL, London, UK. S Edwards is a Senior Lecturer in Bioethics in the UCL Division of Research Strategy and UCL/UCL Hospital Comprehensive Biomedical Centre, London.

\section{References}

1. Haynes JD, Rees G. Decoding mental states from brain activity in humans. Nat Rev Neurosci. 2006; 7:523-534. [PubMed: 16791142]

2. Boly M, et al. Perception of pain in the minimally conscious state with PET activation: an observational study. Lancet Neurol. 2008; 7:1013-1020. [PubMed: 18835749]

3. Giacino JT, et al. The minimally conscious state: definition and diagnostic criteria. Neurology. 2002; 58:349-353. [PubMed: 11839831]

4. Rees G. Neural correlates of the contents of visual awareness in humans. Philos Trans R Soc Lond B Biol Sci. 2007; 362:877-886. [PubMed: 17395576]

5. Rees G, et al. Neural correlates of conscious and unconscious vision in parietal extinction. Neurocase. 2002; 8:387-393. [PubMed: 12499413]

6. Nachev P, Husain M. Comment on "Detecting awareness in the vegetative state". Science. 2007; 315:1221. [PubMed: 17332394]

7. Edwards SJL. The doctrine of double effect is difficult but not impossible to apply. BMJ. 2001; 323:388-391. [PubMed: 11509434]

8. Sandin, P. Better Safe than Sorry: Applying Philosophical Methods to the Debate on Risk and the Precautionary Principle. KTH, Infrastructure; Stockholm: 2004. Theses in Philosophy from the Royal Institute of Technology 